\title{
Essential Thrombocythemia and Acute Myocardial Infarction Treated with Rescue Coronary Angioplasty
}

\author{
Antonio Esteves $\mathrm{F}^{\circ}$, Francisco de Assis Costa, Antonio Augusto Guimarães Lima, \\ Luiz Junya Kajita, Eulógio Emílio Martinez F \\ São Paulo, SP - Brazil
}

\begin{abstract}
A 48-year-old man with essential thrombocythemia suffered an extensive anterior acute myocardial infarction; this is a rare association. A pharmacological thrombolysis was performed, without success. He subsequently underwent successful rescue coronary angioplasty. To our knowledge, there is no other report in the literature relating the triad of essential thrombocythemia, acute myocardial infarction and rescue coronary angioplasty.
\end{abstract}

Essential thrombocythemia (ET) is a primary myeloproliferative disorder in which the platelet count becomes elevated. The predominant clinical manifestations of ET result from hemorrhagic or thromboembolic complications ${ }^{1}$. Factors related to vascular dynamics, such as vanishing platelet thrombi, are likely to play an important role in the genesis of myocardial ischemia and infarction, events rarely found in individuals with $\mathrm{ET}^{2,3}$. In this study, a case of anterior acute myocardial infarction (AMI) diagnosed in a patient with known ET is reported. The patient received thrombolytic therapy, followed by successful rescue coronary angioplasty (RCA).

\section{Case Report}

A 48-year-old white male patient arrived at the Emergency Department (ED) of the Instituto do Coração (INCOR) complaining of chest pain consistent with coronary artery disease, which had started $3 \mathrm{~h}$ before arrival at the ED. At the time of hospital admission, the physical examination showed no abnormal findings, with the exception of high blood pressure $(180 / 120 \mathrm{mmHg})$. The electrocardiogram showed an elevation of the ST segment in leads $\mathrm{D}_{1}, \mathrm{aVL}$, and from $\mathrm{V}_{1}$ to $\mathrm{V}_{6}$, consistent with extensive anterior AMI. Intravenous streptokinase was started with a $3 \mathrm{~h}$ delay (representing the time interval between the development of the symptoms and the beginning of the infusion). There were no clinical criteria for myocardial reperfusion and the patient was classified as group II of the

Instituto do Coração do Hospital das Clínicas - FMUSP

Mailing Address: Antonio Esteves $\mathrm{F}^{\circ}$ - Incor - Serviço de Hemodinâmica - Av. Dr. Enéas C. Aguiar, 44 - 05403-000 - São Paulo, SP
Killip-Kimball heart failure classification. Thus, RCA was started $9 \mathrm{~h}$ after the beginning of the symptoms. The coronary angiography, performed according to the Judkins technique, showed an occlusion of the proximal third of the left anterior descending coronary artery (LAD), without any evidence of atherosclerotic lesions in the remaining arteries (fig. 1). The RCA (balloon) of the LDA was successfully performed. The control angiography showed occlusion of the distal portion of the $2^{\text {nd }}$ marginal branch of the left circumflex artery (M2), as a result of the migration of a thrombus. This vessel was also dilated, with a successful outcome (fig. 2).

There were no incidents during hospitalization. CKMB peaked at $244 \mathrm{U} / \mathrm{l}$ (normal range, up to $10 \mathrm{U} / \mathrm{l}$ ) and the echocardiography performed 3 days after the coronary event showed a value of $59 \%$ for the ejection fraction of the left ventricle, septal hypokinesis, and akinesis of the apical wall.

The patient reported high blood pressure for approximately the previous 20 years and use of amlodipine on an irregular basis. He denied other risk factors for coronary artery disease and reported that, 3 months before, he had suffered from self-limited chest pain with similar characteristics, pain of short duration unrelated to physical or emotional stress. The patient knew he suffered from ET 4 years earlier, when he was diagnosed with it by an expert, based on laboratory findings (platelet count, peripheral blood smear, and platelet aggregation tests) and confirmed by myelogram. He was not in use of any specific medication forET. The platelet count was $1,100,000 / \mathrm{mm}^{3}$, without other significant abnormalities in white blood cell or red blood cell counts. The protein electrophoresis, the urine analysis, the chest films and the abdominal ultrasonography did not show any significant abnormalities. The patient was discharged on the $16^{\text {th }}$ day of hospitalization and was prescribed acetylsalicylic acid, nitrate, beta-blocker, angiotensin-converting enzyme inhibitor and hydroxyurea, $1,500 \mathrm{mg} / \mathrm{d}$.

\section{Discussion}

In many published series, the rate of cardiovascular complications related to the presence of ET ranged from $4 \%$ 


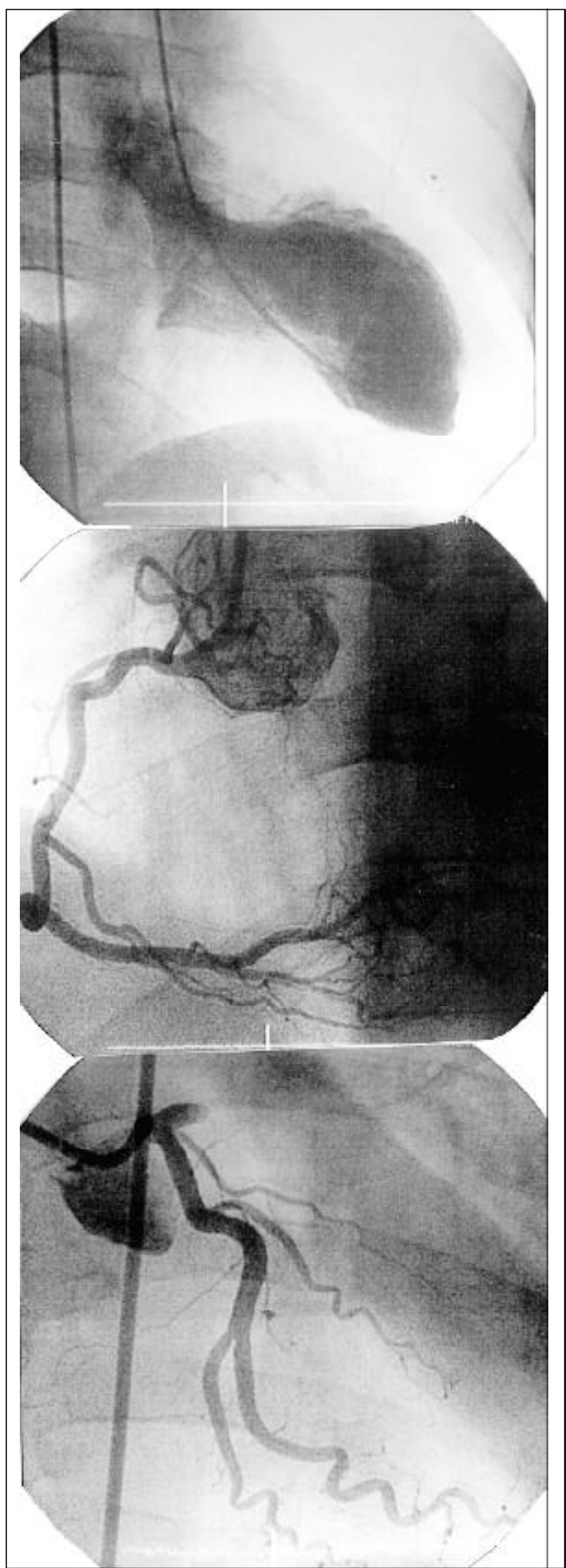

Fig. 1- A) Left ventriculography in RAO, showing marked increase in the enddiastolic volume due to akinesis of the anteroapical wall of the left ventricle; B) right coronary artery in LAO showing normal angiographic aspect; $\mathrm{C}$ ) left coronary artery in posteroanterior (PA) view showing occlusion of the proximal third of the LAD, with a normal angiographic aspect of the circumflex artery. to $21 \%{ }^{4}$. This disorder may lead to the formation of thrombi and acute arterial occlusion, as well as hemorrhagic complications, probably as a result of platelet dysfunction. In both manifestations of the disease, a reduction of platelet aggregation, mainly with adrenaline, is frequently noted ${ }^{1}$. However, when platelet antiaggregating agents are used, as occurred in the present case, these tests lack diagnostic utility. Although rare, sudden death and AMI are complications described in hematologic diseases, such as myeloproliferative disorders characterized by significant changes in the platelet count ${ }^{3}$. When the platelet count exceeds $600,000 / \mathrm{mm}^{3}$, ET may cause coronary thrombosis in different arteries ${ }^{5}$. Possible explanations for this phenomenon include: 1) platelet activation as a result of endothelial injury; 2) prolonged arterial spasm with subsequent thrombosis; 3 ) increased activity of the platelets; 4) changes in the glycoprotein of the platelet granules in patients with ET; and 5) possible selective deficiency of lipoxygenase in individuals with myeloproliferative disorders ${ }^{5}$. In a study involving 55 patients with ET, secondary thrombocytosis, polycythemia vera, chronic granulocytic leukemia, agnogenic myeloid metaplasia and myelodysplastic syndrome, Raman et al ${ }^{6}$ performed, in 43 of these patients, a series of tests to evaluate the hematological profile. These tests included thorough analysis of platelet aggregation, from platelet factor IV, von Willebrand factor and ultrastructural study, to electronic microscopy and bone marrow biopsy. The latter showed that all the patients suffering from ET, polycythemia vera and chronic granulocytic leukemia had a moderate to significant increase of the reticular fibers. It was concluded that $62 \%$ of all patients showed abnormal results to one or more of the inducers employed in the platelet aggregation tests. It was also concluded that different responses in the aggregation tests, levels of platelet factor IV, b-thromboglobulin, factor VIII and fibrinogen, are useful in distinguishing ET from secondary thrombocytosis. Scheffer et $\mathrm{al}^{4}$, reported a series of 7 patients, 6 with ET and one with polycythemia vera; two of them suffered AMI and 5 unstable angina. All the patients under 50 years of age showed normal coronary arteries or one-vessel disease. In this same series, the authors successfully controlled the thrombotic events during 1 to 6 years with the use of aspirin or busulfan, suggesting the ineffectiveness of the therapy with warfarin. The use of hydroxyurea, 500 to $1,500 \mathrm{mg} / \mathrm{d}$, is supported by the literature. These findings are in accordance with the results of the present study in which there were no concurrent atherosclerotic lesions. The absence of spleen enlargement in a patient who did not receive specific therapy for ET, a finding that is quite infrequent, is also of note. On the other hand, considering the fact that the patient was a hypertensive male, almost reaching the $6^{\text {th }}$ decade of life, one must not rule out that the atherosclerotic coronary disease could account for the acute event. However, the apparently healthy condition of the remaining coronary arteries leads one to the assumption that the infarction could have occurred as a primary result of the coagulopathy. To our knowledge, there is no other report in the literature of the triad ET, AMI and RCA. 


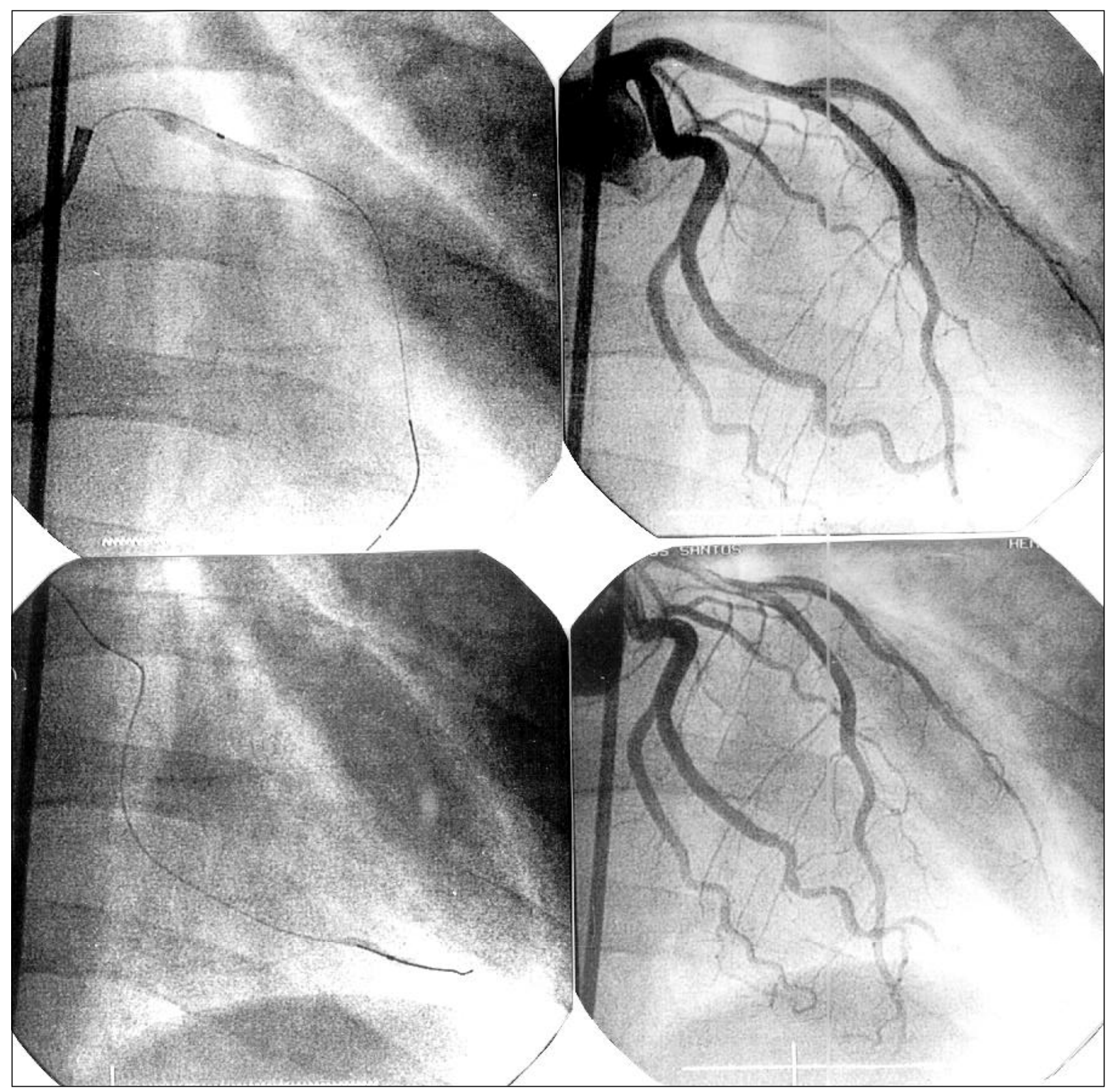

Fig. 2 - A) Insufflated balloon in the proximal portion of the AD; B) contrast medium injection showing a successful procedure in the proximal third of the AD and occlusion of the distal third of the M2; C) Insufflated balloon in the distal portion of M2; D) final result, showing that both dilations were successful.

\section{References}

1. Pick RA, Glover UM, Nanfro JJ, Dubbs WF, Gibbons JA, Vieweg WVR. Acute myocardial infarction with essencial thrombocythemia in a young man. Am Heart J 1983; 106: 406-7.

2. Heggtviet HA. Coronary thrombosis and myocardial infarction. J Am Coll Cardiol 1983; 2:785.

3. Rumoroso JR, Montes PM, Jiménez A, et al. Trombocitosis y enfermedad coronaria. A propósito de un caso. Rev Esp Cardiol 1996; 49: 542-4.
4. Scheffer MG, Michiels JJ, Simoons ML, Roelandt JRTC. Thrombocythemia and coronary artery disease. Am Heart J 1991; 122: 573-6.

5. Douste-Blazy P, Taudou MJ, Delay, et al. Essential thrombocythaemia and recurrent myocardial infarction. Lancet 1984; 2: 992.

6. Raman BKS, Van Slyck EJ, Riddle J, Sawdyk MA, Abraham JP, Saeed SM. Platelet function and structure in myeloproliferative disease, myelodysplastic syndrome and secondary thombocytosis. Am J Clin Pathol 1989; 91: 647-55. 